\title{
Anpassungsverfahren beim gesetzlichen Mindestlohn: Argumente gegen eine politische Lohnfindung
}

\author{
Hagen Lesch $•$ Helena Schneider • Christoph Schröder
}

Angenommen: 4. Juni 2021 / Online publiziert: 6. Juli 2021

(C) Der/die Autor(en) 2021

Zusammenfassung Die Mindestlohnkommission entscheidet alle zwei Jahre über eine Anpassung des gesetzlichen Mindestlohns. Bisher orientierte sie sich dabei nachlaufend an der Tariflohnentwicklung. Das trug dazu bei, dass der Arbeitsmarkt den staatlichen Eingriff in die Lohnfindung gut verkraftete und die Tarifparteien trotz einer Beschränkung ihres Handlungsspielraums weiterhin gewillt sind, die Entgelte autonom auszuhandeln. Der Bundesarbeitsminister kündigte jedoch an, den Mindestlohn rasch auf $12 €$ je Stunde erhöhen zu wollen. Außerdem soll das Anpassungsverfahren beim gesetzlichen Mindestlohn verändert werden und bei der weiteren Entwicklung 60\% des Medianeinkommens (Living Wage) als Referenzgröße dienen. Mit einem solchem Systemwechsel, durch den der Staat dreimal so stark in das Tarifsystem eingreifen würde wie mit der Einführung des Mindestlohns 2015, würde die Tarifautonomie noch deutlicher eingeschränkt. Der Blick auf Frankreich und das Vereinigte Königreich zeigt, dass ein solcher Systemwechsel hin zu einem Living Wage durch Lohnsubventionen flankiert wird. Deshalb müsste bei der Diskussion über einen Systemwechsel beim Mindestlohn in Deutschland auch darüber gesprochen werden, ob die bisherigen Sozialleistungssysteme geändert werden müssen.

Schlüsselwörter Tarifautonomie $\cdot$ Living Wage $\cdot$ Mindestlohnkommission · Frankreich · Vereinigtes Königreich

Dieser Aufsatz basiert auf einem Gutachten im Auftrag der Initiative Neue Soziale Marktwirtschaft.

Helena Schneider $(\square)$

Institut der deutschen Wirtschaft Köln e.V. (IW), Konrad-Adenauer-Ufer 21, 50668 Köln,

Deutschland

E-Mail: helena.schneider@iwkoeln.de 


\title{
Adjusting the statutory minimum wage: arguments against political wage setting
}

\begin{abstract}
Every two years, the Minimum Wage Commission decides how to adjust the statutory minimum wage. Up to now, it has followed the development of collectively agreed wages. This has helped the labor market to cope well with the minimum wage and has ensured that the collective bargaining parties have remained willing to negotiate pay autonomously. Despite these positive outcomes, the Federal Minister of Labor announced to quickly raise the minimum wage to 12 euros per hour. More fundamental, the adjustment procedure for the statutory minimum wage is to be changed. In the medium term, the minimum wage is to be raised to the level of the living wage which is $60 \%$ of the median income. At this level, the state intervention would affect three times more employees as it did with the introduction of the minimum wage in 2015. Thus, the system change towards a living wage would restrict collective bargaining autonomy even more significantly. In France and the United Kingdom, a system change towards a living wage is already completed (France) or on its way (UK). In both countries the system change is flanked by wage subsidies. Therefore, when considering the introduction of a living wage as a statutory minimum wage in Germany, it must be borne in mind that severe changes in the social security system could then also be necessary.
\end{abstract}

Keywords Free collective bargaining $\cdot$ Living wage $\cdot$ Minimum Wage Commission · France · United Kingdom

\section{Einleitung}

Der Gesetzgeber hat Anfang 2015 einen gesetzlichen Mindestlohn eingeführt und dessen Höhe auf 8,50€ festgelegt. Gleichzeitig hat er geregelt, dass die weiteren Anpassungen einer Mindestlohnkommission (MLK) übertragen werden. Die MLK hat sich bei den Anpassungen bisher nachlaufend an der Tariflohnentwicklung orientiert. Inzwischen mehren sich aber Forderungen, diesen Anpassungsprozess einmalig zu ändern. Vorgeschlagen wurde zunächst, den Mindestlohn im Rahmen einer außerordentlichen Anhebung durch die Politik auf $12 €$ je Stunde zu erhöhen (Pusch und Schulten 2019, S. 336). Bundesarbeitsminister Hubertus Heil kündigte im Dezember 2020 an, nicht nur das Anpassungsverfahren ändern, sondern als zusätzliches Kriterium auch das mittlere Einkommen heranziehen $\mathrm{zu}$ wollen. In einem Interview nannte Heil als Orientierungsmarke 60\% des Medianeinkommens (Heil 2020). Ein solcher Lohn wird auch als Living Wage bezeichnet.

Dieser Vorschlag, der im März 2021 durch die Vorlage eines Eckpunktepapiers konkretisiert wurde (BMAS 2021), wird im vorliegenden Beitrag vor dem Hintergrund der bisherigen Arbeit der Mindestlohnkommission kritisch geprüft. Dabei werden auch Erfahrungen aus Frankreich und dem Vereinigten Königreich berücksichtigt, wo die Mindestlöhne deutlich höher sind. In einem ersten Schritt werden die bisherigen Mindestlohnanpassungen bewertet und in einem zweiten wird dargestellt, wie sich der Mindestlohn bei einer Orientierung an $60 \%$ des Medianeinkom- 
mens entwickelt hätte (Kap. 2). Es folgt eine Analyse der Anpassungsprozesse in Frankreich und im Vereinigten Königreich sowie ein Vergleich mit den deutschen Erfahrungen (Kap. 3). Anhand dieses Vergleichs werden abschließend Lehren für die derzeitige politische Debatte in Deutschland gezogen (Kap. 4).

\section{Mindestlohnanpassung im Vergleich}

\subsection{Nachlaufende Orientierung am Tariflohnindex}

Mit der Einführung eines gesetzlichen Mindestlohns in Höhe von 8,50€ je Stunde zum 1. Januar 2015 durch das ,Gesetz zur Regelung eines allgemeinen Mindestlohns (Mindestlohngesetz - MiLoG)“ griff der Staat erstmals seit dem Bestehen der Bundesrepublik Deutschland direkt in die Lohnfindung und in die Tarifautonomie ein. Bisher beschränkten sich staatliche Eingriffe auf die Erstreckung von Tarifverträgen im Wege der Allgemeinverbindlichkeit. Bei diesem Prozedere, das insbesondere im Rahmen des Arbeitnehmer-Entsendegesetzes genutzt wird, wird den Tarifparteien aber eine aktivere Rolle zugewiesen als beim gesetzlichen Mindestlohn. Sie werden selbst aktiv, um mit Hilfe des Staates tarifliche Branchenmindestlöhne auf nichttarifgebundene Betriebe zu erstrecken. Außerdem sehen die tariflichen Mindestlöhne eine Differenzierung zwischen den betroffenen Branchen vor. Die Regelungen zum gesetzlichen Mindestlohn sehen keine solche Differenzierung vor, außerdem werden die Tarifparteien nicht freiwillig aktiv - sie werden vom Staat verpflichtet. Um ein Mindestmaß an Autonomie zu wahren, wurde gemäß $\S 4$ Abs. 1 MiLoG eine ständige Mindestlohnkommission eingerichtet, die über die Anpassung der Höhe des Mindestlohns befindet. Ihr Vorschlag wird von der Regierung geprüft und rechtlich umgesetzt. Die Kommission besteht aus einem Vorsitzenden und sechs weiteren stimmberechtigten Mitgliedern. Diese werden paritätisch auf Vorschlag der Arbeitgeberverbände und Gewerkschaften besetzt. Zu diesen insgesamt sieben stimmberechtigten Mitgliedern kommen zwei beratende Mitglieder aus Kreisen der Wissenschaft, die kein Stimmrecht haben.

Das MiLoG verpflichtet die MLK gemäß $§ 9$ Abs. 1 dazu, den Mindestlohn alle zwei Jahre anzupassen. Dabei hat sie im Rahmen einer „Gesamtabwägung“ zu prüfen, welche Mindestlohnhöhe zu einem angemessenen Mindestschutz der Arbeitnehmerinnen und Arbeitnehmer beiträgt, faire Wettbewerbsbedingungen ermöglicht und Beschäftigung nicht gefährdet. Zudem hat sich die MLK bei der Festsetzung des Mindestlohns nachlaufend an der Tariflohnentwicklung zu orientieren ( $\$ 9$ Abs. 2 Satz 3 MiLoG). Diese Vorgabe entsprach ausdrücklich dem Wunsch beider Tarifparteien (Riechert und Nimmerjahn 2017, S. 212f.). Man kann diese Koppelung zwischen Mindestlohn- und Tariflohnentwicklung als ein Regelbindungselement interpretieren (Lesch und Schröder 2020a, S. 166). Eine Regelbindung hat zwei wichtige Vorteile, die umso eher greifen, je strikter sie eingehalten wird: Erstens stellt sie sicher, dass der Mindestlohn nicht zu sehr in das Tarifgeschehen eingreift; zweitens vermeidet sie, dass Mindestlohnanpassungen nicht zum ständigen Gegenstand politischer Auseinandersetzungen werden. 
Die im MiLoG vorgesehene nachlaufende Orientierung an der Tariflohnentwicklung begründet keinen mechanischen Automatismus zwischen Tariflohnentwicklung und Höhe der Anpassung (Wissenschaftliche Dienste 2016, S. 9). Insofern handelt es sich um keine Regelbindung im strikten Sinne. Sie lässt der MLK im Rahmen ihrer Gesamtabwägung Spielraum. Das MiLoG schreibt auch nicht explizit vor, wie die Tariflohnentwicklung zu messen ist (Riechert und Nimmerjahn 2017, S. 213). Die MLK hat dies daher autonom in einer Geschäftsordnung (GO) geregelt, die im Januar 2016 verabschiedet wurde. Sie zieht den Tarifindex ohne Sonderzahlungen auf Basis der Stundenverdienste in den beiden vorhergehenden Kalenderjahren des Statistischen Bundesamts heran ( $\$ 3$ Abs. 1 Geschäftsordnung MLK). Zudem sieht die GO der MLK in $\S 3$ Abs. 2 vor, dass die MLK bei ihren Anpassungsbeschlüssen nur mit einer Zwei-Drittel-Mehrheit von der nachlaufenden Orientierung an der Tariflohnentwicklung abweichen darf (Wissenschaftliche Dienste 2016, S. 4 und 8).

\subsection{Bewertung der Anpassungen 2016 bis 2020}

Der Mindestlohn ist im Kontext einer Gesamtabwägung anzupassen. Die dort genannten Ziele können in einem Spannungsverhältnis zueinanderstehen. Das Ziel, einen existenzsichernden Mindestlohn durchzusetzen, kann zum Beispiel mit dem Ziel der Beschäftigungsstabilität in Konflikt geraten. Es ist daher sinnvoll, die Anpassung des Mindestlohns einer Kombination aus Regelbindung (nachlaufende Orientierung am Tarifindex) und diskretionärer Anpassung zu unterwerfen. Stellt die MLK fest, dass eine nachlaufende Orientierung in Bezug auf die Gesamtabwägung wenig sinnvoll ist, kann sie den diskretionären Spielraum nutzen. Dieser Spielraum ist ein autonomer Spielraum, der eine flexible Anpassung des Mindestlohns möglich macht. Er unterscheidet sich von einem Anpassungsmechanismus, bei dem aus dem politischen Raum Vorgaben gemacht werden.

Die MLK hat bisher dreimal über eine Anpassung des Mindestlohns entschieden:

- Im Juni 2016 beschloss sie, den Mindestlohn ab Januar 2017 von 8,50€ auf $8,84 €$ je Stunde anzuheben (MLK 2016, S. 1).

- Der zweite Beschluss vom Juni 2018 sah eine Anhebung in zwei Stufen vor. Mit Jahresbeginn 2019 wurden 9,19€ und mit Jahresbeginn 2020 dann 9,35€ je Stunde vorgesehen (MLK 2018, S. 1).

- Beim dritten Anpassungsbeschluss vom Juni 2020 wurden Mindestlohnanpassungen in vier Stufen beschlossen (MLK 2020, S. 1): 9,50€ ab Januar 2021, 9,60€ ab Juli 2021, 9,82€ ab Januar 2022 und 10,45€ ab Juli 2022.

Beim dritten Beschluss hatte die Kommission die Auswirkungen der Corona-Pandemie ebenso zu berücksichtigen wie die politische Debatte über einen Mindestlohn von $12 €$ je Stunde. Aufgrund des rezessiven Einflusses der Pandemie schlug sie für 2021 nur einen Inflationsausgleich vor. Die reguläre (sich rechnerisch aus der Entwicklung des Tarifindexes ergebende) Erhöhung auf 9,82€ je Stunde wurde um ein Jahr auf Anfang 2022 verschoben. Die MLK (2020, S. 2) geht davon aus, dass dann wieder eine „Rückkehr auf das Niveau des Bruttoinlandsprodukts von vor der Pandemie zu erwarten“ sei. Der für Juli 2022 vorgesehene vierte Schritt auf 10,45€ je Stunde ist der politischen Debatte geschuldet. Er stellt eine rein diskretionäre An- 
passung dar, die sich nicht durch die Entwicklung des Tarifindexes begründen lässt. Die MLK (2020, S. 2) betont allerdings, dass die Tarifparteien durch die frühzeitige Ankündigung die Möglichkeit hätten, die Mindestlohnentwicklung bei der Fortentwicklung von Tarifverträgen zu berücksichtigen. Fasst man den Gesamtzeitraum von zwei Jahren zusammen, entspricht die Belastung in etwa einer Belastung, die sich aufgrund einer rein regelgebundenen Anpassung auf 9,82€ ab Januar 2021 ergeben hätte.

Um zu überprüfen, inwieweit die MLK in ihren drei bisherigen Anpassungsbeschlüssen der im MiLoG verankerten Regelbindung gefolgt ist, werden die Anpassungen dem hypothetischen Szenario einer starren regelgebundenen Anpassung gegenübergestellt. Bei einer regelgebundenen Anpassung wird davon ausgegangen, dass der Mindestlohn alle zwei Jahre angehoben wird und dieser Anhebung gemäß $\S 3$ Abs. 1 GO die Entwicklung des Tarifindexes ,in den beiden vorhergehenden Kalenderjahren“" zugrunde liegt. Da die Anpassung unterjährig beschlossen wird, ist offen, welchen Zeitraum dies genau betrifft. Zum Vergleich wurden zwei regelgebundene Anpassungen berechnet:

1. Bei der regelgebundenen ex-ante Anpassung ist die Tariflohnentwicklung zwischen Juni des Jahres t-3 und Juni des Jahres t-1 Grundlage einer Anpassung zum Januar des Jahres t. Der Juni wurde anstelle des Julis als Startzeitpunkt gewählt, weil der Tarifindex zum Monatsultimo ausgewiesen wird und der 30. Juni näher am 1. Juli liegt als der 31. Juli. Der ersten Anhebung zum Januar 2017 liegt demnach der Anstieg des Tarifindexes zwischen Juni 2014 und Juni 2016 zugrunde.

2. Bei einer regelgebundenen ex-post Anpassung wird bei einer Anpassung zum Januar des Jahres t immer die Tariflohnentwicklung zwischen Dezember des Jahres t-3 und Dezember des Jahres t-1 zugrunde gelegt. Der ersten Anhebung zum Januar 2017 liegt hier der Anstieg des Tarifindexes zwischen Dezember 2014 und Dezember 2016 zugrunde. Ex post bedeutet hier, dass die der MLK-Entscheidung zugrundeliegende zweijährige Entwicklung des Tarifindexes immer erst im Nachhinein bekannt ist.

In Abb. 1 sind diese beiden regelgebundenen Anpassungen der tatsächlichen Mindestlohnanpassung gegenübergestellt. Zusätzlich wurde auch die Entwicklung des Tarifindexes abgetragen. Für die Jahre 2021 und 2022 wurde die Tariflohndynamik unter der Annahme fortgeschrieben, dass die Tarifverdienste 2021 um 1,6\% und 2022 um 2,5\% steigen. Die ex-post Anpassung führt automatisch dazu, dass die Treppenfunktion alle zwei Jahre an die stetig ansteigende Linie der Tariflohnentwicklung stößt. Es ist erkennbar, dass auch die regelgebundene ex-ante Anpassung dem Tarifindex strikt folgt. Lediglich Anfang 2021 liegt sie etwas über dem Tarifindex. Die tatsächliche Anpassung des Mindestlohns bleibt bis Ende 2020 hinter der Entwicklung der regelgebundenen ex-post Anpassung und bis Mitte 2021 hinter der Entwicklung der regelgebundenen ex-ante Anpassung zurück. Durch die diskretionäre Anpassung zum Juli 2022 übersteigt die tatsächliche Mindestlohnanpassung erstmals den regelgebundenen Anpassungspfad und die prognostizierte Entwicklung des Tarifindexes.

Zusammenfassend lässt sich festhalten, dass die MLK im Rahmen ihrer Gesamtabwägung stets das Gesamtbild im Blick hatte. Dabei hat sie der Regelbindung 


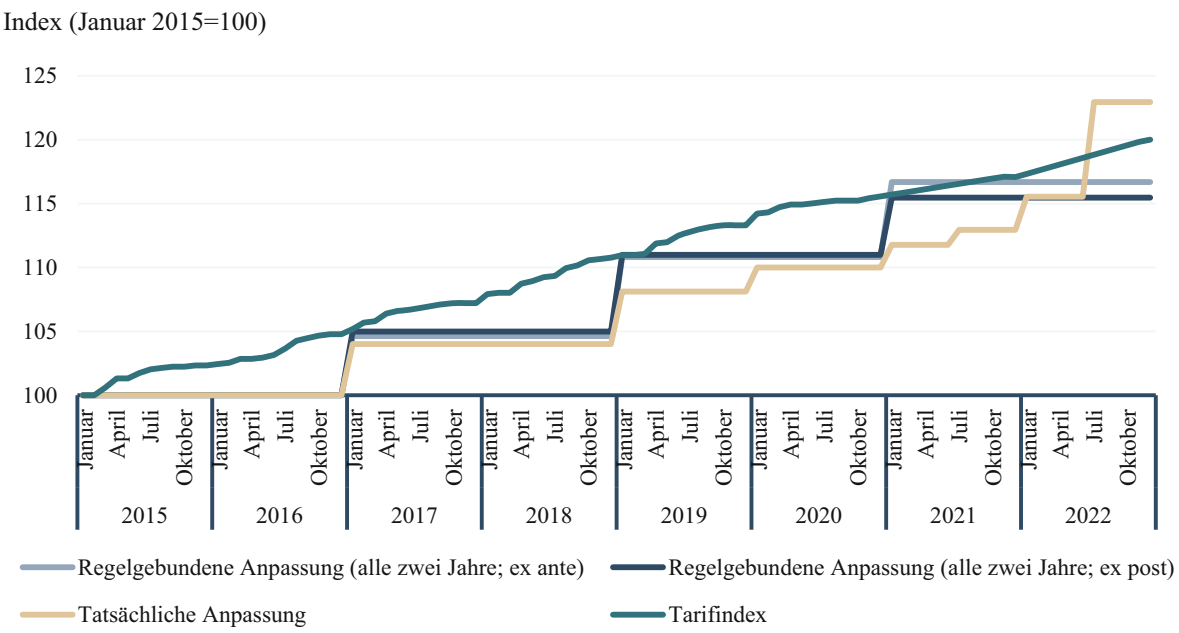

Abb. 1 Regelgebundene und tatsächliche Mindestlohnanpassungen. Index (Januar 2015=100). (Quellen: Statistisches Bundesamt; Mindestlohnkommission; eigene Berechnungen)

Rechnung getragen, aber nicht rein technisch, sondern politisch abwägend. Mit der 2020 beschlossenen Erhöhung auf 10,45€ schießt die MLK erstmals über den Pfad einer regelgebundenen Anpassung hinaus.

\subsection{Mindestlohn und Living Wage}

Die Debatte über einen Mindestlohn von $12 €$ in Deutschland wird flankiert von einer europäischen Debatte. Die Europäische Kommission hat Ende Oktober 2020 einen Richtlinienentwurf über angemessene Mindestlöhne in der Europäischen Union veröffentlicht (Europäische Kommission 2020). Dieser sieht vor, die Anpassung des Mindestlohns stärker an ,das allgemeine Niveau der Bruttolöhne und ihre Verteilung" (Europäische Kommission 2020, S. 14) zu koppeln. Als positiv bewertet wird im Entwurf das Szenario, die Mindestlöhne auf $60 \%$ des (Brutto-)Medianlohns der Vollzeitbeschäftigten anzuheben. Dieser Wert wird oft auch als Living Wage oder existenzsichernder Lohn bezeichnet. Im Folgenden wird untersucht, wie hoch der Living Wage in Deutschland wäre und wie er sich bis 2024 entwickeln könnte.

Der Medianlohn je Stunde wird in Deutschland explizit nur im Rahmen der Verdienststrukturerhebung (VSE) ausgewiesen (Statistisches Bundesamt 2020). Aus der VSE liegen aber nur Angaben für 2014 und 2018 vor. Im Jahr 2014 lag der Median-Stundenlohn für Vollzeitbeschäftigte nach dieser Quelle bei 17,35€ und 2018 bei $19,09 €$ je Stunde. Der daraus abgeleitete Living Wage hätte 2014 demnach bei $10,41 €$ und 2018 bei $11,45 €$ gelegen. Mit Hilfe anderer Verdienstindikatoren und mit Prognosen für die Verdienstentwicklung lässt sich der Living Wage für die Jahre zwischen 2014 und 2018 interpolieren und bis 2024 fortschreiben. Für das Jahr 2019 lässt sich dies anhand von Daten der Bundesagentur für Arbeit für den Median-Monatsverdienst machen, die einen Anstieg von 2,9\% ausweisen (Bundesagentur für Arbeit 2020). Damit hätte der Living Wage 2019 bei 11,79€ je Stunde 
In Euro je Stunde

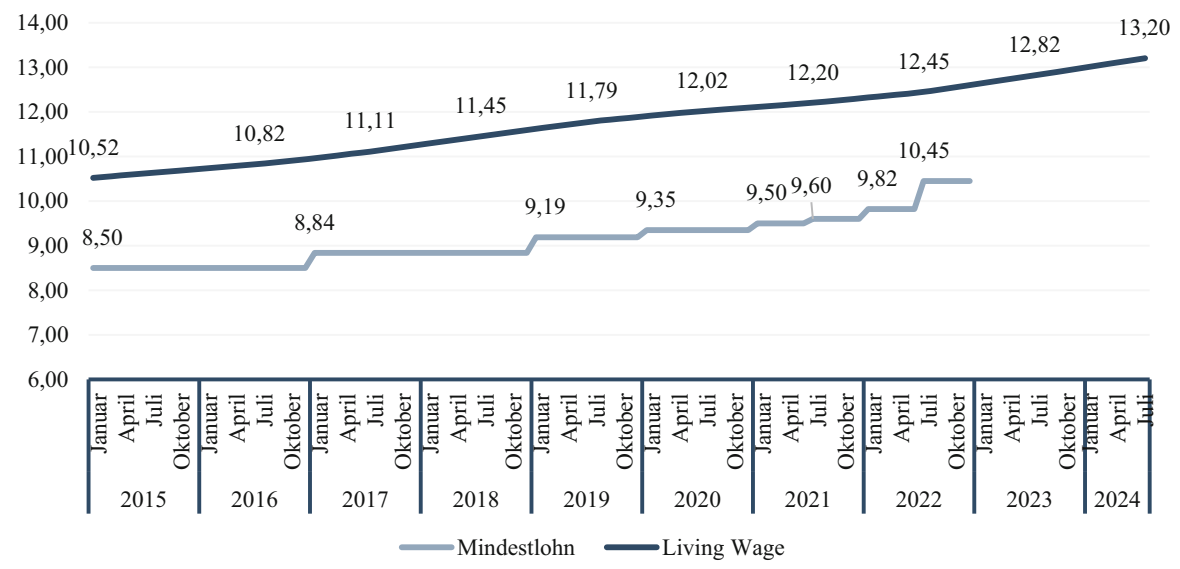

Abb. 2 Gesetzlicher Mindestlohn und Living Wage (in Euro je Stunde). Living Wage: 60\% des Medians der Stundenverdienste von Vollzeitbeschäftigten. (Quellen: Bundesagentur für Arbeit; Statistisches Bundesamt; eigene Berechnungen)

gelegen. Für 2020 wird ein Anstieg von Medianlohn und Living Wage um 2,0\% angenommen, was in etwa der Dynamik der Effektivverdienste und der Tarifverdienste einschließlich Sonderzahlungen entspricht. Daraus errechnet sich für 2020 ein Wert von 12,02€. Damit wäre die 12-Euro-Marke im Falle einer Orientierung des Mindestlohns am Living Wage bereits 2020 überschritten worden. Mit jedem weiteren Anstieg des Medianlohns würde der politisch diskutierte Wert von $12 €$ hinter einen Living Wage zurückfallen.

Im Juni 2022 wird die MLK über die Höhe des Mindestlohns für die Jahre 2023 und 2024 entscheiden. Aufgrund der diskutierten Kopplung des Mindestlohns an das Medianeinkommen wird im Folgenden auch die Höhe des Living Wage für die Jahre bis 2024 geschätzt. Hierzu sind einige Annahmen zu treffen, die unter dem Vorbehalt einer großen Unsicherheit über die weitere wirtschaftliche Entwicklung stehen, die durch die Corona-Pandemie verursacht wird. Aufgrund der derzeit schlechten Ertragssituation der Unternehmen und der vorerst weiter schwierigen konjunkturellen Lage wird davon ausgegangen, dass sich der Living Wage 2021 um 1,5\%, 2022 um $2,0 \%$ und 2023 sowie 2024 um jeweils 3,0\% erhöht. Damit würde der Living Wage 2021 zunächst auf $12,20 €$ steigen und 2022 bei $12,45 €$ liegen (Abb. 2). Im Jahr 2023 würde er gut $12,80 €$ und 2024 dann 13,20€ betragen. Im Mittel der Jahre 2023 und 2024 ergeben sich somit $13 €$. Gegenüber dem für das zweite Halbjahr 2022 beschlossenen Mindestlohn von 10,45€ wäre dies ein Anstieg von mehr als $24 \%$.

Abschließend stellt sich die Frage, wie sich der Mindestlohn entwickelt hätte, wenn er sich schon seit 2015 am Medianlohn orientiert hätte. Zwischen 2015 und 2022 stieg der Mindestlohn von 8,50€ auf 10,14€ (Durchschnitt aus 9,82€ im ersten Halbjahr und 10,45€ im zweiten Halbjahr) - also um 19,2\%. Wäre der Mindestlohn ab Januar 2015 an die Entwicklung des Medianlohns beziehungsweise des Living Wage gebunden gewesen, hätte sich bis 2022 unter den getroffenen Annah- 
men ein Anstieg von 18,0\% ergeben. Damit steigt der gesetzliche Mindestlohn nach der bisherigen Anpassungspraxis bis Ende 2022 schneller, als er im Falle einer Orientierung am Living Wage gestiegen wäre. Bis zum zweiten Halbjahr 2022 errechnet sich für den gesetzlichen Mindestlohn sogar ein Anstieg von 22,9\%. Diese Gegenüberstellung deutet an, dass von einer Umstellung auf einen anderen Verdienstindikator - wie vom Bundesarbeitsminister vorgeschlagen - vermutlich langfristig kein deutlich beschleunigter Anstieg des Mindestlohns zu erwarten wäre. Im Falle der Einführung eines Living Wage würde sich dieser Anstieg indes auf einem deutlich erhöhten Niveau fortsetzen.

Eine Einführung eines Mindestlohns in Höhe von $12 €$ hätte - ebenso wie die Etablierung eines Living Wage in Höhe von $60 \%$ des Medianeinkommens - spürbare Auswirkungen auf die Tarifverhandlungen. Einer Auswertung von mehr als 40 Tarifbranchen zufolge lagen Anfang 2019 etwa 20\% aller Lohngruppen in Tarifverträgen unter der Schwelle von $12 €$ je Stunde (Pusch und Schulten 2019, S. 337). Damit wäre die Eingriffsintensität des Mindestlohns in das Tarifsystem etwa dreimal so hoch wie 2015, als gut $6 \%$ aller tariflichen Entgeltgruppen unter dem gesetzlichen Niveau von 8,50€ je Stunde lagen (Bispinck et al. 2020, S. 25). Eine Angabe zur Anzahl der betroffenen Beschäftigten ist nicht möglich, da keine Informationen zur Besetzung der Tariflohngruppen vorliegen (vgl. auch die Auswertung in: Statistisches Bundesamt 2019, S. 9 ff.). Dabei ist zu berücksichtigen, dass es nach der Mindestlohneinführung bis Ende 2017 eine Übergangsphase gab, die den Tarifparteien Zeit zur Anpassung ihrer Tarifstrukturen einräumte. Insgesamt hat das Tarifsystem den damaligen Eingriff gut überstanden. Eine Analyse des Tarifgeschehens ausgewählter Branchen zeigt zwar, dass Tariflöhne durch den Mindestlohn zeitweise verdrängt und das Lohngefüge zum Teil gestaucht wurde. Die Tarifparteien haben sich aber letztlich an die neuen Rahmenbedingungen anpassen können (Bispinck et al. 2020).

\section{Internationale Erfahrungen}

In der Europäischen Union (EU) sind gesetzliche Mindestlöhne in den meisten Mitgliedstaaten eine schon lange geübte Praxis (Lübker und Schulten 2021). Vor diesem Hintergrund stellt sich die Frage, was sich aus den Erfahrungen anderer Länder lernen lässt. Von den vier größten Volkswirtschaften in der EU haben neben Deutschland auch Frankreich und das inzwischen aus der EU ausgetretene Vereinigte Königreich einen gesetzlichen Mindestlohn, während Italien darauf verzichtet. Aus einer Analyse des französischen und britischen Modells können daher wichtige Erkenntnisse für die Diskussion in Deutschland gewonnen werden. Der Blick auf diese beiden Länder hat zudem den Vorteil, dass ein etatistisch geprägtes Land mit einem eher liberal geprägten Land verglichen wird.

\subsection{Der Mindestlohn in Frankreich}

In Frankreich hat der Mindestlohn eine lange Tradition. Bereits 1950, also unmittelbar nach dem Ende des Zweiten Weltkriegs, kam es zur Einführung des sogenannten 
SMIG (,salaire minimum interprofessionnel garanti“). Dieser „,berufsübergreifend garantierte Mindestlohn“ sollte allen Beschäftigten ein Existenzminimum sichern. 20 Jahre später wurde der SMIG jedoch grundlegend reformiert und wurde zum SMIC (,salaire minimum interprofessionnel de croissance"), der die fortan angestrebte Wachstumsorientierung bereits mit im Namen trug (Mesch 2011, S. 574f.). Denn der 1970 eingeführte SMIC soll nicht allein eine untere Einkommensgrenze und ,ein angemessenes Lohnniveau zur Erhaltung der Kaufkraft gewähren“, sondern auch Niedrigverdienern die Teilhabe am wirtschaftlichen Erfolg garantieren (Deutscher Bundestag 2018, S. 4f.).

Bei der vorgeschriebenen Anpassung des Mindestlohns zum 1. Januar eines jeden Jahres spielen die Entwicklung der Verbraucherpreise und die durchschnittliche Entwicklung der realen Stundenlöhne eine entscheidende Rolle. Diesbezüglich liegt der jährlichen Mindestanpassung ein Indexierungsmechanismus zugrunde. So muss die Erhöhung des Mindestlohns mindestens die Inflationsrate seit der letzten Erhöhung ausgleichen. Zudem muss die Anpassung preisbereinigt mindestens halb so hoch sein wie die Steigerungsrate bei den durchschnittlichen realen Stundenlöhnen. Eine tripartistische Institution mit Regierungs-, Gewerkschafts- und Arbeitgebervertretern - „Commission Nationale de la Négociation Collective“ (CNNC) - legt zu bevorstehenden Erhöhungen eine Empfehlung vor, die aber rechtlich nicht bindend ist (Cette und Wasmer 2012, S. 4 ff.). Zusätzlich wird die Regierung seit 2009 von einem mit Wissenschaftlerinnen und Wissenschaftlern besetzten Expertengremium, der ,groupe d'experts sur le SMIC“, beraten. Festgeschrieben wird der Mindestlohn per Dekret durch die Regierung (Lesch et al. 2014, S. 8 f.).

Steigt die Inflation im Verlauf eines Jahres über 2\%, wird der SMIC automatisch unmittelbar nach der Veröffentlichung des relevanten Preisindexes auch unterjährig entsprechend angepasst. Unabhängig von der Lohnentwicklung und der Inflation kann der Mindestlohn zudem zu jeder Zeit durch die Regierung angepasst werden. Bei einem solchen „,coup de pouce“ handelt es sich um eine außerordentliche Mindestlohnerhöhung, die über die im Gesetz festgeschriebene Mindestanpassung hinausgeht. Eine solche Erhöhung wird in unregelmäßigen Abständen von der Regierung beschlossen, damit die Lücke zwischen SMIC und Durchschnittslohn nicht kontinuierlich ansteigt. So wird dem Grundgedanken des SMIC Rechnung getragen, auch Niedrigverdiener an der wirtschaftlichen Entwicklung des Landes teilhaben zu lassen (Mesch 2011, S. 575).

Der französische Mindestlohn gilt grundsätzlich für alle nichtselbstständig Beschäftigten in Frankreich. Ausnahmeregelungen bestehen lediglich für Jugendliche unter 18 Jahren mit weniger als sechs Monaten Berufserfahrung, Auszubildene unter 26 Jahren und Praktikanten. So darf der Stundenlohn von unter 17-Jährigen beispielsweise nicht weniger als $80 \%$ des SMIC-Niveaus betragen und der von 17Jährigen nicht unter $90 \%$ des SMIC liegen (Französische Botschaft in Deutschland 2020).

Nachdem 1970 der ursprünglich eingeführte SMIG durch den SMIC ersetzt wurde, kam es zu einem dynamischen Wachstum des Mindestlohns bis Anfang der 1980er-Jahre. In dieser Phase wuchs der SMIC schneller als das Durchschnittseinkommen. Gründe dafür waren der neu eingeführte Indexierungsmechanismus sowie eine rege Verabschiedung diskretionärer Anhebungen. So entwickelte sich der 
SMIC - wie durch die Reformen beabsichtigt - von einer bloßen Lohnuntergrenze zu einem lohnpolitischen Instrument zur Reduzierung von Ungleichheit. Vor dem Hintergrund wirtschaftlicher Probleme wie Inflation und Arbeitslosigkeit flachte das SMIC-Wachstum im Verlauf der 1980er bis Mitte der 1990er-Jahre ab (Gautié und Laroche 2018, S. 3).

Kurz vor der Jahrtausendwende verzeichnete der französische Mindestlohn wieder einen stärkeren Anstieg. Zum einen wirkte sich ein höheres Wirtschaftswachstum positiv auf die Mindestlohnhöhe aus. Zum anderen wurde aber auch die reguläre Wochenarbeitszeit von 39 auf 35 Stunden abgesenkt. Trotz dieser Arbeitszeitverkürzung sollte es zu keinem Einkommensverlust bei Mindestlohnempfängern kommen. Diese Zielsetzung führte dazu, dass Beschäftigte, die bereits vor der Arbeitszeitreform angestellt waren, trotz vier Stunden weniger Arbeit pro Woche ihr ursprüngliches Einkommen entsprechend einer 39-Stunden-Woche erhielten. Nach der Reform eingestellte Arbeitnehmerinnen und Arbeitnehmer wurden hingegen gemäß einer 35Stunden-Woche bezahlt. Um diese Ungleichbehandlung von Mindestlohnempfängern aufzuheben, kam es bis 2006 zu einer Harmonisierung, die de facto einen deutlichen Anstieg des Mindestlohns bedeutete. Seitdem sind die Regierungen wieder zu einer eher zurückhaltenden Mindestlohnpolitik zurückgekehrt, was sich auch in einem selteneren Gebrauch von außerordentlichen Mindestlohnanpassungen niederschlägt (Gautié und Laroche 2018, S. 4).

Zum 1. Januar 2020 lag der französische Mindestlohn bei 10,15€. Damit rangiert Frankreich bei einem europäischen Vergleich der Mindestlohnhöhen direkt hinter Luxemburg auf Platz zwei (Schulten und Lübker 2020, S. $121 \mathrm{f}$.). Wird der sogenannte Kaitz-Index, also das Verhältnis zwischen Mindestlohn und mittlerem Lohn eines Landes, aus dem Jahr 2019 betrachtet, ist Frankreich sogar europäischer Spitzenreiter. So betrug der Mindestlohn 2019 61,4\% des Medianlohns und 49,6\% des Durchschnittslohns (OECD 2021).

In Frankreich fielen 2016 mit gut 10\% vergleichsweise viele Beschäftigte in die Gruppe der Mindestlohnempfänger. Das hat neben der im europäischen Vergleich hohen absoluten und relativen Mindestlohnhöhe noch weitere Gründe. Bei der Berechnung und Kontrolle des SMIC werden keine Zuschläge, Sonderzahlungen, Prämien oder Alterszulagen berücksichtigt. Folglich kommen viele Lohnbestandteile bei der als Mindestlohnempfänger deklarierten Gruppe noch dazu, sodass die tatsächliche Bezahlung vieler Beschäftigter dieser Gruppe deutlich über dem Mindestlohnniveau liegen dürfte (Französische Botschaft in Deutschland 2020).

Darüber hinaus werden Unternehmen für Beschäftigte mit einem Gehalt bis zur 1,6-fachen Höhe des Mindestlohns umfassende Reduktionen bei den Sozialabgaben gewährt (Lesch et al. 2014, S. 9). Eingeführt wurden diese Erleichterungen 1993, um der damals hohen Arbeitslosigkeit in der Gruppe der Geringqualifizierten und unter Jugendlichen entgegenzuwirken. Für die angespannte Arbeitsmarktlage in diesen Beschäftigungsgruppen wurde auch das im internationalen Vergleich hohe französische Mindestlohnniveau verantwortlich gemacht, das bei geringqualifizierten Beschäftigten zu einem Missverhältnis zwischen Produktivität und Arbeitskosten führte. Da eine Absenkung des Mindestlohns politisch nicht gewollt war, wurden die Arbeitskosten für Niedriglohnbeschäftigte durch eine Reduktion der Sozialbeiträge gesenkt (OECD 1995, S. 56 ff.). Diese staatliche Subventionierung von Niedriglöh- 
nen soll negativen Beschäftigungseffekten vorbeugen, erhöht aber auch den Anreiz, Arbeitnehmerinnen und Arbeitnehmer in der Niedriglohngruppe zu beschäftigen (Mesch 2011, S. 576). So können die Kostenvorteile für Unternehmen auch zu einer Mindestlohnfalle für Beschäftigte werden. Denn Unternehmen könnten Lohnerhöhungen scheuen, wenn diese zusätzlich zu einem Wegfall der Reduzierungen bei den Sozialabgaben führen. Zudem verursacht die Reduktion der Sozialabgaben eine erhebliche finanzielle Belastung des französischen Haushalts (Schulten und Müller 2020, S. 47).

Da der französische Mindestlohn in ein komplexes Konstrukt aus unterschiedlichen Arbeitsmarktinstitutionen eingebettet ist, kann empirisch nicht eindeutig geklärt werden, mit welchen Beschäftigungseffekten er einhergeht. Während einige Studien keine Auswirkungen des Mindestlohns auf das Beschäftigungsniveau finden, konstatieren andere Studien insbesondere für Jugendliche negative Beschäftigungseffekte (Lesch et al. 2014, S. 9 f.; Gautié und Laroche 2018). Cahuc und Carcillo (2012) können zeigen, dass die Reduzierung der Sozialabgaben für Niedriglohnverdiener mögliche negative Beschäftigungseffekte abfedert und eine Kürzung dieser Subventionierungen mit erheblichen Arbeitsplatzverlusten verbunden wäre.

\subsection{Der Mindestlohn im Vereinigten Königreich}

Im Vereinigten Königreich wurde der gesetzliche Mindestlohn im Jahr 1999 eingeführt (Lesch et al. 2014, S. 10f.). Damit erfüllte die Labour Party, die 1998 erstmals wieder seit 18 Jahren die Regierung stellte, ein langjähriges Wahlversprechen. Hintergrund hierfür war nicht zuletzt die stark gesunkene Tarifbindung (Schulten und Müller 2020, S. 52). Rechtliche Grundlage ist der National Minimum Wage Act 1998. Festgelegt wird der Mindestlohn von der Regierung, die alle Details regeln kann. Dabei sollen keine Unterscheidungen nach Region, Sektor des Arbeitsmarktes, Unternehmensgröße oder Beruf gemacht werden (§ 3). Nach dem Alter darf nur dann differenziert werden, wenn die Person jünger als 26 Jahre ist. Aktuell gibt es vier Stufen: Auszubildende, 16- bis 17-Jährige, 18- bis 20-Jährige und 21- bis 24-Jährige (gültig ab April 2021 für 21- bis 22-Jährige, zwischen 2010 und 2016 ab 21 Jahren, 1999 bis 2009 ab 22 Jahren). Diese Mindestlöhne für Jüngere werden von der LPC separat festgelegt (Francis-Devine 2020, S. 7). Ebenfalls gesetzlich bindend ist der 2016 eingeführte National Living Wage (NLW), der zunächst für alle Beschäftigten ab 25 Jahren und ab April 2021 bereits ab einem Alter von 23 Jahren gilt. Ausnahmen vom Mindestlohn sind für neueingestellte Beschäftigte sowie Personen in Aus- und Weiterbildung oder in Beschäftigungsprogrammen möglich. Fischer mit Gewinnbeteiligungen als Einnahmequelle und ehrenamtlich Tätige sind explizit ausgeschlossen ( $\$ 43$ f.). Der Regierung wird zudem das Recht eingeräumt, den Personenkreis, für den Ausnahmen gelten können, zu erweitern ( $\$ 4)$.

Die Regierung sollte bei der Einführung des Mindestlohns die bereits 1997 eingerichtete Low Pay Commission (LPC) konsultieren (Lesch et al. 2014, S. 10). Dies kann sie auch bei allen weiteren Fragen tun, die den Mindestlohn betreffen $(\S 5$ und $\S 6$ ). Macht sie von diesen Möglichkeiten Gebrauch und hält sich nicht an die von der LPC beschlossenen Empfehlungen, muss die Regierung in beiden Häusern des Parlaments einen Bericht vorlegen, in dem sie die Abweichungen begründet. Die 
LPC soll bei den Berichten, die sie zu verfassen hat, Vertreter der Arbeitgeberorganisationen und der Gewerkschaften befragen. Bei ihren Empfehlungen hat die LPC die Auswirkungen ihrer Vorschläge auf die Wirtschaft im Vereinigten Königreich und auf deren Wettbewerbsfähigkeit zu berücksichtigen ( $§ 7)$. Die LPC selbst setzt sich aus insgesamt neun Mitgliedern zusammen, die von der Regierung berufen werden. Jeweils drei sollen gewerkschaftsnah oder arbeitgebernah sein oder aus der Wissenschaft stammen. Das britische Mindestlohngesetz betont somit die Rolle der Regierung bei der Festlegung des Mindestlohns und gibt der LPC eher eine beratende Funktion. Diese hat gleichwohl Gewicht, da die Regierung Abweichungen von den LPC-Empfehlungen vor dem Parlament vertreten muss.

In der Praxis wird der Mindestlohn im Vereinigten Königreich jährlich angepasst. Etwa ein halbes Jahr bevor eine Erhöhung ansteht, wird die LPC beauftragt, einen Bericht zu erstellen. Der Auftrag an die LPC war dabei bis 2015 fast gleichlautend: Sie sollte eine Mindestlohnhöhe festsetzen, die so vielen niedrig entlohnten Beschäftigten wie möglich hilft, ohne dabei jedoch das Beschäftigungsniveau zu verringern und die Wirtschaft zu schädigen (LPC 2016, S. vii). Die Abstimmung in der Kommission war bisher einstimmig (Schulten und Müller 2020, S. 53). Ihre Empfehlungen wurden von der Regierung in aller Regel unverändert übernommen und in eine Rechtsverordnung umgesetzt. Eine Ausnahme stellt der Mindestlohn für Auszubildende dar, den die Regierung im Jahr 2015 um $21 \%$ heraufsetzte (BIS 2015, S. 5).

Seit 2016 nimmt die Politik jedoch erheblichen Einfluss auf die Entwicklung des Mindestlohns. Schon mit der Vorlage des Haushalts im Sommer 2015 kündigte die Regierung zu einem konjunkturell sehr günstigen Zeitpunkt an, im April 2016 für alle Beschäftigten, die 25 Jahre und älter sind, einen sogenannten National Living Wage (NLW) einzuführen. Für die unter 25-Jährigen sollte auch weiterhin der bisherige Mindestlohn mit seinen getroffenen Altersstaffelungen gelten. Bei seiner Einführung betrug der NLW 7,20£. Das waren 50 Pence mehr als der alte Mindestlohn, der für die Altersgruppe der 21- bis 24-Jährigen weiterhin Bestand hatte. Der Wert des NLW entsprach rund 55\% des Median-Stundenlohns der Berechtigten (alle Beschäftigte, die mindestens 25 Jahre alt sind). Bis zum Jahr 2020 sollte er auf $60 \%$ des Medians der Berechtigten erhöht werden. Auch in der aktuellen Debatte um einen Living Wage in der EU gilt dieser Wert als eine Art Benchmark in formaler Analogie zum Schwellenwert für Armutsgefährdung. Allerdings bezieht er sich zumeist auf die Vollzeitbeschäftigten (Europäische Kommission 2020). Proklamiertes Ziel der britischen Regierung war ein Regimewechsel von einem System mit niedrigen Löhnen, hohen Steuern und hohen Sozialausgaben zu einem Regime mit hohen Löhnen, niedrigen Steuern und niedrigen Sozialausgaben. Zu diesem Wechsel sollte auch der NLW beitragen, zumal die Regierung den im Vereinigten Königreich weit überdurchschnittlichen Anteil der Niedriglohnbeschäftigten beklagte (HM Treasury 2015, S. 31).

Der NLW war Teil einer politischen Gesamtstrategie. Seine Einführung im Jahr 2016 geschah ohne vorherige formale Konsultation der LPC und seine Ankündigung im Sommer 2015 kam für Gewerkschaften, Arbeitgeber und den LPC gleichermaßen überraschend (Sellers 2017, S. 795). Die LPC sah in der Einführung des NLW die größte Änderung im Mindestlohnregime seit seiner Einführung (LPC 2016, S. vii). 
Zwar seien mit dem National Minimum Wage Act 1998 keine Ziele für die Politik festgelegt worden, die Debatten hätten aber gezeigt, dass es um mindestens drei Ziele ging: Die Ausbeutung der Beschäftigung zu vermeiden, die Löhne zu erhöhen und die Politik aus der Setzung des Mindestlohns herauszuhalten und stattdessen Empfehlungen von einer unabhängigen Expertenkommission einzuholen (ebenda). Die LPC betonte, ihr übergeordneten Ziel sei es gewesen, Niedrigstlöhne zu verhindern, ohne dabei Jobs zu gefährden oder die Wirtschaft insgesamt zu beschädigen. In der Verfolgung dieses Ziels sei sie erfolgreich gewesen. Mit der Einführung des NLW werde die strategische Zielsetzung verändert. In einer Stellungnahme stell die Kommission dazu fest (LPC 2016, S. vii f.):

- Der Grundsatz der LPC, keine signifikanten Arbeitsplatzverluste zu verursachen, würde zumindest aufgeweicht. So schätzte eine Behörde des Finanzministeriums, dass die Arbeitslosigkeit im Jahr 2020 mit NLW um 60.000 Personen oder 0,2 Prozentpunkte höher liegen würde als ohne NLW (Office for Budget Responsibility 2015, S. 204 f.).

- Da der NLW erst für Beschäftigte in einem Alter von 25 Jahren gilt, würde mit dem NLW faktisch eine fünfte Mindestlohnstufe eingeführt. Das bisher für alle Erwachsene ab 21 Jahren geltende Mindestlohnniveau gelte nur noch für die Gruppe der 21- bis 24-Jährigen. Dies mache es schwieriger, Altersdiskriminierungen zu vermeiden. Zudem sah sich die LPC für den bisherigen gesetzlichen Mindestlohn in seinen vier Altersstufen weiterhin an eine strengere Auslegung der Beschäftigungsneutralität gebunden (LPC 2019, S. v). Dies erschwere zusätzlich die Aufgabe, den NLW mit dem Mindestlohn der 21- bis 24-Jährigen in Einklang $\mathrm{zu}$ bringen.

- Anders als bisher für den gesetzlichen Mindestlohn gäbe es für den NLW ein mittelfristiges politisches Ziel, nämlich diesen bis 2020 auf $60 \%$ des Medianlohns anzuheben (BIS 2015, S. 7).

Diese Punkte zeigen, dass die LPC die Einführung des NLW durchaus kritisch sah. Denn zum einen wurde der Grundsatz der Beschäftigungsneutralität aufgegeben, zum anderen wurde der LPC durch die Nennung eines mittelfristigen Zielwerts eine passive Rolle zugewiesen und der Pfad der Mindestlohnentwicklung anders als zuvor aktiv von der Politik vorgegeben. Die Regierung beteuerte zwar, dass die LPC weiterhin eine Schlüsselrolle in der Festsetzung des Mindestlohns spiele und auch über das Tempo der Heraufsetzung des NLW beraten solle, nahm ihr aber faktisch den Handlungsspielraum. Denn auch die Sätze des Mindestlohns für junge Erwachsene lassen sich nicht unabhängig von dem NLW festsetzen, da bei zu großen Unterschieden ein Anreiz bestünde, über 24-Jährige durch 21- bis 24-Jährige zu ersetzen oder die jüngeren Erwachsenen bevorzugt einzustellen.

Zur Entlastung der kleinen Firmen wurde die erst 2014 eingeführte Employment Allowance (EA) im Jahr 2016 von $2000 £$ auf $3000 £$ pro Betrieb und Jahr erhöht. Mit der EA können die Arbeitgeberbeiträge zur Sozialversicherung (National Insurance) abgesenkt werden. Bei seiner Einführung sei die EA so hoch gewesen, dass der Arbeitgeber für insgesamt vier Beschäftigte, die Vollzeit zum gesetzlichen Mindestlohn arbeiten, keine Sozialbeiträge zahlen musste. Nun sei dies für vier Beschäftigte, die den NLW erhalten, möglich (Office for Budget Responsibility 2015, S. 34). Au- 
Berdem wurde der Beitragssatz zur Körperschaftssteuer um 1 Prozentpunkt gesenkt. Inzwischen ist die EA weiter auf $4000 £$ erhöht worden.

Das Ziel, den Mindestlohn auf 60\% des Medians der Berechtigten anzuheben, wurde erreicht und die entsprechenden Schritte dorthin wurden von der LPC vorgeschlagen. Nach dem Wunsch der Regierung soll der NLW auch nach 2020 kräftig steigen. Bereits im Herbst 2018 erklärte die Regierung bei der Vorstellung ihres Haushalts, den Mindestlohn in Relation zum Medianlohn noch weiter erhöhen zu wollen und damit die Zahlung von Niedriglöhnen zu beenden (HM Treasury 2018, S. 79). Dies bedeutet, dass der NLW von 60 auf knapp $67 \%$ des Medianlohns der Berechtigten erhöht werden soll. Der Einfluss auf Beschäftigung und Arbeitsmarkt soll dabei berücksichtigt werden. Bereits mit der Bekanntgabe des neuen Ziels, also rund zweieinhalb Jahre vor Inkrafttreten der ersten Stufe auf dem Weg zur formalen Abschaffung der Niedriglohnbeschäftigung, verkündete die Regierung, dass sie eine Weisung an die LPC für die Jahre nach 2020 geben würde (ebenda).

Die LPC schlug dann im Herbst 2019 vor, den NLW in Zukunft ab 21 Jahren geltend $\mathrm{zu}$ machen und damit wieder zu einem System mit vier, statt jetzt fünf Altersklassen zurückzukehren. Hierzu soll in einem Zwischenschritt der NLW ab 2021 für Beschäftigte ab 23 Jahre gelten (LPC 2019, S. xiii). Die Regierung legte daraufhin das Ziel fest, bis 2024 den NLW auf zwei Drittel des Medianlohns der Berechtigten zu erhöhen, und die Altersgrenze im Jahr 2021 auf zunächst 23 Jahre und bis 2024 weiter auf 21 Jahre abzusenken. Der zweite Termin ist nur als Zielwert formuliert. Gleichzeitig installiert die Regierung eine Notbremse (,Emergency Break"): Sie weist die LPC an, auf auftauchende Risiken aufmerksam zu machen und falls es die ökonomische Situation erfordert, der Regierung zu empfehlen, ihre Ziele oder den Zeitrahmen zu revidieren. Die LPC wird zudem aufgerufen, ihre Methode, mit der sie den Einfluss des NLW evaluiert, darzulegen und weiterzuentwickeln (BEIS 2020, S. 3). Damit geht die Regierung auf die Wünsche der LPC nach größeren Freiräumen und höherer Flexibilität ein. Das vorsichtigere Vorgehen ist aber auch vor dem Hintergrund der unsicheren ökonomischen Entwicklung durch den Brexit und die Corona-Krise zu sehen. Dies spiegelt sich auch darin, dass die Erhöhung des NLW mit 2,2\% für 2021 - knapp über der erwarteten Inflationsrate eher niedrig ausfällt und bereits eine deutliche Unterschreitung des Zielpfads beschreibt. Gleichwohl rät die LPC - auch wegen der aktuell unsicheren Datenlage noch nicht zu einer Verschiebung des Ziels, die Niedriglohnbeschäftigung bis 2024 zu beenden (LPC 2020, S. xxi).

Diese kurze Darstellung zeigt, dass sich das System der Mindestlohnfindung im Vereinigten Königreich deutlich gewandelt hat. Während die Regierung bis 2015 die Entscheidung über die Mindestlohnhöhe weitgehend der LPC überlassen hat, gibt jetzt die Regierung das Tempo vor, räumt der LPC seit 2020 aber wieder eine stärkere Mitwirkung ein.

Der Mindestlohn im Vereinigten Königreich startete auf einem im internationalen Vergleich niedrigen Niveau. Er betrug 42,3\% des Medianlohns der Vollzeitbeschäftigten gegenüber damals 61,5\% in Frankreich. In Deutschland lag das relative Niveau bei der Einführung des Mindestlohns im Jahr 2015 bei $48 \%$. Die Entwicklung des Mindestlohns verlief dann in vier Phasen (Francis-Devine 2020, S. 7 f.). 
- Der Mindestlohn wurde zu einem niedrigen Niveau eingeführt und verlief dann in den ersten 18 Monaten parallel zur Preisentwicklung.

- Nachdem die Forschung in dieser Frühphase keine negativen Effekte der Mindestlohneinführung auf Beschäftigung und Wirtschaft feststellen konnte, wurde der Mindestlohn bis 2007 auch real kräftig erhöht: Die Kaufkraft des Mindestlohns stieg zwischen 2000 und 2007 um $34 \%$.

- Während der langen Rezession und der erst allmählichen Erholung der Wirtschaft gab der Mindestlohn zwischen 2008 und 2013 in realer Rechnung kontinuierlich nach.

- Schließlich setzte 2014 ein erneuter realer Anstieg ein, auf dem ab 2016 der NLW als zusätzliche Stufe aufgesetzt wurde. Im Jahr 2020 lag der NLW real fast $61 \%$ höher als der Mindestlohn in der Altersklasse ab 22 Jahre bei seiner Einführung im Jahr 1999.

Auch in Relation zum Medianlohn ist der Mindestlohn über die Zeit kräftig gestiegen. Gemessen am Kaitz-Index der OECD, der den Mindestlohn in Relation zum Medianlohn aller Vollzeitbeschäftigten setzt, ergibt sich ein Anstieg von 42,3\% im Jahr 1999 auf 55,1\% im Jahr 2019, wobei sich der letztgenannte Wert auf den NLW bezieht. Bis zum Systemwechsel 2015 stieg der Kaitz-Index bereits auf 48,6\%. Selbst in der wirtschaftlichen Schwächephase zwischen 2008 und 2013 gab es einen Anstieg von 1,1 Prozentpunkten. Auch ohne große Einflussnahme der Regierung ist der Mindestlohn also kontinuierlich stärker gestiegen als der mittlere Lohn. Bei der Betrachtung des Kaitz-Index der OECD ist zu berücksichtigen, dass sich dieser nur auf Vollzeitarbeitnehmer bezieht. Diese haben aber deutlich höhere Stundenlöhne als Teilzeitbeschäftigte. Wenn die Briten Zielwerte für den NLW in Relation zum Medianlohn festsetzen, beziehen sich diese auf alle Berechtigten und schließen daher die Teilzeitbeschäftigten ein. Daher entspricht das Ziel, den NLW auf knapp $67 \%$ (zwei Drittel) des Medianlohns bis 2024 zu erhöhen (dann für alle Beschäftigten ab 21 Jahren) einem OECD-Kaitz-Index von rund 60\%. Damit bliebe das Vereinigte Königreich, selbst wenn das Anpassungsziel für 2024 erreicht wird, noch knapp unter dem Niveau von Frankreich (Cribb et al. 2019, S. 5).

Aus Arbeitgebersicht sind nicht die Relationen des Bruttolohns, sondern die Unterschiede bei den Arbeitskosten entscheidend. Zugute kommt den britischen Arbeitgebern daher ein Freibetrag bei der Sozialversicherung von $792 £$. Dadurch ist bei NLW-Empfängern, die in Vollzeit arbeiten, ein größerer Teil des Lohns beitragsfrei als bei den übrigen Vollzeitbeschäftigten. Für kleine Firmen mit sehr wenigen Beschäftigten fallen wegen der oben beschriebenen Employment Allowance ebenso wie für Teilzeitbeschäftigte im günstigsten Fall für den Arbeitgeber überhaupt keine Sozialversicherungsbeiträge an. Durch diese Mechanismen ist für die britischen Arbeitgeber ein OECD-Kaitz-Index von $60 \%$ im Idealfall der Beitragsfreiheit in etwa so gut tragbar wie ein Wert von $55 \%$ in einem Land, in dem die effektiven Arbeitgeberbeitragsätze für die Sozialversicherung für alle Beschäftigten gleich hoch sind. 


\subsection{Vergleich europäischer Mindestlohnregime}

Um die Mindestlohnregime zwischen Deutschland, Frankreich und dem Vereinigten Königreich vergleichend einordnen zu können, müssen neben der institutionellen Ausgestaltung des Mindestlohnregimes auch flankierende Maßnahmen und Funktionslogiken des zugrundeliegenden Arbeits- und Sozialsystems betrachtet werden.

Auf die kürzeste Mindestlohngeschichte blickt Deutschland zurück. Hier wurde die Lohnuntergrenze erst 2015 und damit erheblich später als in Frankreich (1950) und im Vereinigten Königreich (1999) eingeführt. Im Unterschied zu diesen beiden Ländern gibt es in Deutschland keinen Living Wage, da der deutsche Mindestlohn weder direkt noch indirekt an die Entwicklung des Medianeinkommens gekoppelt ist und deutlich darunter liegt. Indem er vor unangemessen niedrigen Löhnen schützen soll, hat er auch eine andere Zielsetzung.

Das Verfahren zur Festlegung der Mindestlohnhöhe weist einige Parallelen, aber auch grundlegende Unterschiede auf (Tab. 1). In allen drei Ländern erarbeitet ein Beratungsgremium Vorschläge dazu, wie die Mindestlohnerhöhung aussehen könnte. Und in allen Mindestlohnregimen finden sich in diesem Gremium Vertreter der Tarifparteien. In Frankreich werden darüber hinaus auch politische Akteure (drei Minister sowie der Präsident des Sozialsenats) hinzugezogen, während das Vereinigte Königreich sowie Deutschland zusätzlich auf den Rat von Wissenschaftlern zurïckgreifen. Im Vereinigten Königreich sind die Wissenschaftler stimmberechtigt, in Deutschland kommt ihnen lediglich eine beratende Funktion zu. Die Kommissionen bestehen in Deutschland und im Vereinigten Königreich jeweils aus neun, in Frankreich aus insgesamt 40 Mitgliedern.

Nicht nur die Zusammensetzung, sondern auch die Rolle des Bratungsgremiums und damit auch die Rolle der Tarifparteien, die diese Gremien hauptsächlich besetzen, ist in den drei Ländern unterschiedlich definiert. Die deutsche MLK hat dabei die weitreichendsten Kompetenzen und genießt die größte Autonomie. Bezüglich der Mindestlohnhöhe hat sie nicht nur ein Vorschlagsrecht, sondern faktisch sogar ein Vorschlagsmonopol. Die jeweilige Regierung kann die von der MLK geforderte Erhöhung nur annehmen oder ablehnen, aber keine eigenen Vorschläge unterbreiten. Das MiLoG sieht keine Umsetzung regierungsseitiger Vorschläge vor. In Frankreich und im Vereinigten Königreich stellen die Kommissionen hingegen beratende Organe dar, deren Vorschläge zwar Gewicht haben, von der Regierung jedoch angepasst oder ergänzt werden können. Während der deutschen Regierung eher eine passive Rolle in Form eines Ausführungsorgan zufällt, kommt den Regierungen der Partnerstaaten eine deutlich aktivere Rolle zu. Sie sind nicht nur endgültige Entscheidungsinstanz, sondern haben zudem große diskretionäre Spielräume.

Schließlich unterscheiden sich die drei betrachteten Länder erheblich hinsichtlich der Regelbindungs-Komponenten. In Frankreich liegt den jährlichen Mindestlohnanpassungen ein starrer, im Gesetz verankerter Indexierungsmechanismus zugrunde, der sich an der Inflation und der allgemeinen Lohnentwicklung orientiert. In Deutschland ist hingegen die Entwicklung der Tariflöhne entscheidend. Im Unterschied zu Frankreich handelt es sich hierbei jedoch um eine Orientierungsgröße, denn es ist kein automatischer Mechanismus im Sinne einer Eins-zu-eins-Übertragung festgeschrieben. Das beinhaltet (zumindest theoretisch) nicht nur einen Spiel- 
Tab. 1 Vergleich europäischer Mindestlohnregime. (Quellen: OECD; eigene Darstellung)

\begin{tabular}{|c|c|c|c|}
\hline & Frankreich & Vereinigtes Königreich & Deutschland \\
\hline Bezeichnung & $\begin{array}{l}\text { Salaire minimum } \\
\text { interprofessionnel de } \\
\text { croissance (SMIC) }\end{array}$ & $\begin{array}{l}\text { National Minimum Wage } \\
\text { und National Living Wage } \\
\text { (NLW) }\end{array}$ & Mindestlohn \\
\hline $\begin{array}{l}\text { Einführung } \\
\text { (Systemwechsel } \\
\text { hin zu Living } \\
\text { Wage) }\end{array}$ & $1950(1970)$ & 1999 (2016) & 2015 \\
\hline $\begin{array}{l}\text { Ausrichtung/ } \\
\text { Intention }\end{array}$ & Living Wage & Living Wage & Lohnuntergrenze \\
\hline Beratungsgremium & $\begin{array}{l}\text { Commission Natio- } \\
\text { nale de la Négociati- } \\
\text { on Collective }\end{array}$ & Low Pay Commission & Mindestlohnkommission \\
\hline $\begin{array}{l}\text { Zusammensetzung } \\
\text { des Beratungs- } \\
\text { gremiums }\end{array}$ & $\begin{array}{l}\text { Je } 18 \text { Vertreter von } \\
\text { Gewerkschafts- und } \\
\text { Arbeitgeberseite } \\
3 \text { Minister } \\
\text { Präsident des Sozial- } \\
\text { senats }\end{array}$ & $\begin{array}{l}\text { Je } 3 \text { Vertreter von Gewerk- } \\
\text { schaften, Arbeitgebern und } \\
\text { Wissenschaft } \\
\text { Alle stimmberechtigt }\end{array}$ & $\begin{array}{l}\text { Je } 3 \text { Vertreter von Ge- } \\
\text { werkschafts- und Arbeit- } \\
\text { geberseite } \\
\text { Vorsitzender } \\
2 \text { Wissenschaftler ohne } \\
\text { Stimmrecht }\end{array}$ \\
\hline $\begin{array}{l}\text { Rolle des Be- } \\
\text { ratungsgre- } \\
\text { miums/der } \\
\text { Tarifparteien }\end{array}$ & $\begin{array}{l}\text { Beratende Funk- } \\
\text { tion mit Gewicht/ } \\
\text { Vorschlagsrecht } \\
\text { innerhalb starrer } \\
\text { Vorgaben }\end{array}$ & $\begin{array}{l}\text { Beratende Funktion mit } \\
\text { Gewicht/Vorschlagsrecht }\end{array}$ & $\begin{array}{l}\text { Vorschlagsrecht und } \\
\text { Vorschlagsmonopol }\end{array}$ \\
\hline $\begin{array}{l}\text { Rolle der Re- } \\
\text { gierung }\end{array}$ & $\begin{array}{l}\text { Aktiv, Gestaltungs- } \\
\text { und Entscheidungs- } \\
\text { organ }\end{array}$ & $\begin{array}{l}\text { Aktiv, Gestaltungs- und } \\
\text { Entscheidungsorgan }\end{array}$ & $\begin{array}{l}\text { Passiv, Entscheidungsor- } \\
\text { gan; Regierung kann nur } \\
\text { über Annahme oder Ab- } \\
\text { lehnung des Vorschlags } \\
\text { der Mindestlohnkommis- } \\
\text { sion entscheiden }\end{array}$ \\
\hline Anpassungsturnus & $\begin{array}{l}\text { Jährlich zum 01.01.; } \\
\text { zusätzlich unterjäh- } \\
\text { rige Anpassungen } \\
\text { jederzeit möglich }\end{array}$ & Jährlich zum 01.04 & $\begin{array}{l}\text { Beschluss alle zwei Jah- } \\
\text { re; Beschluss kann un- } \\
\text { terjährige Anpassungen } \\
\text { vorsehen }\end{array}$ \\
\hline $\begin{array}{l}\text { Regelbindungs- } \\
\text { elemente }\end{array}$ & $\begin{array}{l}\text { Indexierung an Infla- } \\
\text { tion und allgemeiner } \\
\text { Lohnentwicklung } \\
\text { Sofortige Anpas- } \\
\text { sung, wenn Inflation } \\
\text { über 2\% }\end{array}$ & Keine & $\begin{array}{l}\text { Nachlaufende Orientie- } \\
\text { rung an der Tariflohnent- } \\
\text { wicklung }\end{array}$ \\
\hline $\begin{array}{l}\text { Diskretionärer } \\
\text { Spielraum }\end{array}$ & $\begin{array}{l}\text { „coup de pouce“: } \\
\text { außerordentliche } \\
\text { Mindestlohnerhö- } \\
\text { hung durch Re- } \\
\text { gierung jederzeit } \\
\text { möglich }\end{array}$ & $\begin{array}{l}\text { Diskretionäres System } \\
\text { Vorgabe von mittelfristig } \\
\text { zu erreichenden Zielgrößen } \\
\text { durch Regierung mit jährli- } \\
\text { chem Auftrag der Regierung } \\
\text { an die Kommission }\end{array}$ & $\begin{array}{l}\text { Diskretionärer Spielraum } \\
\text { der Kommission bei } \\
\text { Zwei-Drittel-Mehrheit } \\
\text { Kein diskretionärer Spiel- } \\
\text { raum der Regierung }\end{array}$ \\
\hline $\begin{array}{l}\text { Bedeutung des } \\
\text { Tarifsystems }\end{array}$ & Gering & Gering & Hoch \\
\hline $\begin{array}{l}\text { Subventionierung } \\
\text { von Mindest- } \\
\text { löhnen }\end{array}$ & $\mathrm{Ja}$ & $\mathrm{Ja}$ (für kleine Unternehmen) & Nein \\
\hline $\begin{array}{l}\text { Kaitz-Index } \\
(2019)\end{array}$ & 61,4 & 55,1 & 48,2 \\
\hline
\end{tabular}


Mindestlöhne in Prozent der Medianlöhne von Vollzeitbeschäftigten

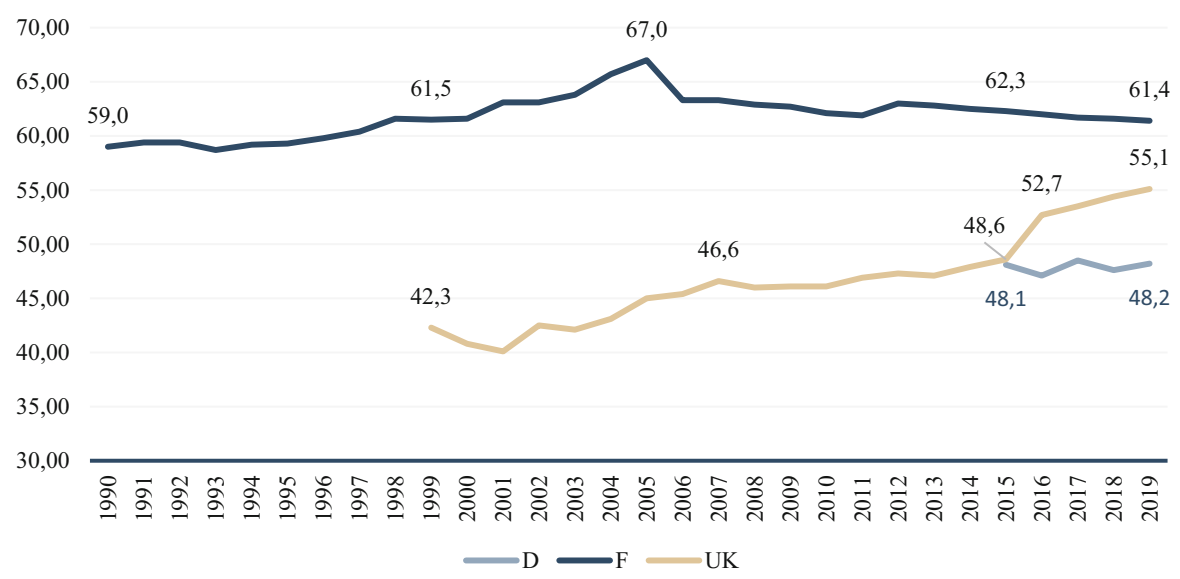

Abb. 3 Entwicklung des Kaitz-Index in Deutschland, Frankreich und im Vereinigten Königreich. Mindestlöhne in Prozent der Medianlöhne von Vollzeitbeschäftigten. D, F: ab 18 Jahre; UK bis 2009 ab 22 Jahre, 2010 bis 2015 ab 21 Jahre, ab 2016 ab 25 Jahre (National Living Wage). (Quelle: OECD)

raum nach oben, sondern auch nach unten. Zudem kann die MLK mit einer ZweiDrittel-Mehrheit von der Orientierung an der Tariflohnentwicklung abweichen. In der Praxis räumt zwar auch das französische Modell viel diskretionären Spielraum ein, da die Regierung durch einen sogenannten ,coup de pouce“ den Mindestlohn jederzeit außerordentlich anheben kann. Den Spielraum gibt es aber nur nach oben, nicht nach unten. Im Vereinigten Königreich finden sich keine gesetzlich festgelegten Regelbindungselemente. Damit ist der diskretionäre Spielraum dort formal am größten. Da der Regierung in Deutschland bisher keine vergleichbaren diskretionären Spielräume zur Verfügung stehen, kann sie allenfalls durch eine Änderung des MiLoG eingreifen, wie es derzeit politisch diskutiert wird.

Hinsichtlich des Kaitz-Indexes ist das Mindestlohnniveau in Frankreich am höchsten, gefolgt vom Vereinigten Königreich (Abb. 3). Im Jahr 2019 lag der Kaitz-Index in Frankreich bei 61,4\% und damit über dem Niveau eines Living Wage. Das Vereinigte Königreich kam auf einen Wert von $55,1 \%$, ist also noch etwas vom einem Living Wage entfernt. Deutschland weist mit 48,2\% zu Frankreich einen Abstand von gut 13 Prozentpunkten auf. Die hohen Kosten des Mindestlohns für Unternehmen werden in diesen beiden Ländern jedoch zumindest teilweise durch Erleichterungen bei den Sozialabgaben abgefangen. So werden die Arbeitgeberbeiträge für die Sozialversicherung in Frankreich für niedrige Löhne deutlich abgesenkt. Bei Jahresverdiensten auf Niveau des SMIC beträgt die sogenannte „Réduction générale des cotisations patronales“ (ehemals: Réduction Fillon) maximal $32 \%$ des Bruttoentgelts. Sie läuft knapp unterhalb des Medianlohns aus. Für einen Beschäftigten, der über ein mittleres Einkommen verfügt, fallen für den Arbeitgeber einschließlich anderer Kostenpositionen Lohnnebenkosten von gut $53 \%$ des Bruttolohns an. Dieser Satz wird für SMIC-Empfänger auf $21 \%$ reduziert. Daraus ergibt sich, dass bei einem Kaitz-Index bezogen auf den Bruttolohn von 61,4, wie ihn die OECD 
für Frankreich im Jahr 2019 ausweist, die Arbeitskosten des SMIC-Empfängers nur 48,6\% des Medianlohn-Beschäftigten ausmachen. Die Kosten für die Reduzierung der Arbeitgeberbeiträge bei niedrigen Löhnen sind nicht genau bekannt. In der Arbeitskostenerhebung 2016 wird der Anteil der gesamten Lohnsubventionen mit 1,9\% des Arbeitnehmerentgelts ausgewiesen. Mit diesem Prozentsatz ergibt sich für 2019 ein Wert von 23,6 Mrd. €.

Auch im Vereinigten Königreich ist die Zusatzkostenquote für den Mindestlohnempfänger niedriger als für einen Beschäftigten, der den Medianlohn erhält. Dies ergibt sich durch einen allgemeinen Arbeitgeberfreibetrag für die Sozialversicherung und durch die Employment Allowance, mit der die Beitragslast von Kleinunternehmen reduziert wird (s. Abschn. 3.2). Der angestrebte Kaitz-Index von ungefähr $60 \%$ nach OECD (zwei Drittel nach nationaler Definition) entspricht daher im günstigsten Fall gut $55 \%$ bei den Arbeitskosten, wenn die Arbeitgeber keine Sozialversicherungsbeiträge zahlen müssen. Die Ausgaben des Staates für die Employment Allowance sind im Haushalt nicht explizit ausgewiesen, lassen sich aber aus den im Haushalt projizierten Be- und Entlastungen für die Einzelmaßnahmen annähernd errechnen. Es ergibt sich für 2020 ein Betrag von 2,5 Mrd. Pfund oder 2,9 Mrd. $€$. Hinzu kommen noch die impliziten Kosten durch die Freibeträge bei der Sozialversicherung.

In Deutschland variieren die Beitragssätze der Arbeitgeber zur Sozialversicherung dagegen nicht nach der Lohnhöhe. Die Relation der Arbeitskosten des MindestlohnEmpfängers zu den Arbeitskosten des Medianlohn-Beschäftigten entspricht daher auch in etwa der Relation bei den Bruttolöhnen und damit dem Kaitz-Index. Dieser lag 2019 nach OECD-Angaben bei 48,2 und dürfte bis zur zweiten Jahreshälfte 2022 noch um gut 3 Prozentpunkte ansteigen. Damit zeigt sich, dass das Arbeitskostenverhältnis des Mindestlohn-Empfängers in Relation zum Medianlohnempfänger in Deutschland schon heute in etwa genauso hoch ist wie in Frankreich.

\section{Schlussfolgerungen}

Der Vergleich der Mindestlohnregime zwischen Frankreich, dem Vereinigten Königreich und Deutschland zeigt, dass dem Mindestlohn in Deutschland eine andere Funktionslogik zugrunde liegt als in den beiden anderen Ländern. Entscheidender Unterschied ist dabei seine fehlende Ausrichtung als Living Wage. Entsprechend liegt das deutsche Mindestlohnniveau gemessen am Kaitz-Index deutlich unter dem der beiden europäischen Partnerstaaten. Vor diesem Hintergrund fordert der Bundesarbeitsminister eine Änderung des Anpassungsmechanismus. Zusätzlich zum Kriterium der Tariflohnentwicklung soll eine Orientierung am Medianeinkommen Teil des Regelwerks werden. Sollten dabei $60 \%$ des Medians festgelegt werden, käme dies einem Systemwechsel hin zu einem Living Wage gleich.

Aus den in Frankreich und dem Vereinigten Königreich gesammelten Erfahrungen lassen sich Hürden aufzeigen, die im Falle eines solchen Strategiewechsels überwunden werden müssten und zu einer kritischen Bewertung dieses Vorschlags führen. Zunächst fällt auf, dass der angestrebte Systemwechsel hin zu einem existenzsichernden Lohn in Deutschland kurzfristiger nach Einführung des Mindestlohns 
als in den Vergleichsländern vollzogen werden soll. Frankreich und das Vereinigte Königreich gingen erst 15 bis 20 Jahre nach der Einführung des gesetzlichen Mindestlohns zu einem Living Wage über. In Deutschland ist der Mindestlohn noch nicht lange eingeführt. Auch wenn er sich insgesamt als Instrument bewährt hat, befindet er sich in seinem sechsten Jahr immer noch in einer Etablierungsphase, zumal die seit seiner Einführung bestehende konjunkturelle „Schönwetterperiode“ einem markanten Wirtschaftseinbruch gewichen ist. Momentan ist nicht verlässlich abschätzbar, welche wirtschaftlichen Folgen die nach wie vor unbewältigte CoronaPandemie noch nach sich ziehen kann. In einer Phase, in der sich der Mindestlohn erstmals in einer Wirtschaftskrise bewähren muss, sollte erst einmal abgewartet werden, bevor leichtfertig ein Systemwechsel angeschoben wird. Würde er rasch umgesetzt, bestünde das Risiko, dass der vorgeschlagene Eingriff viele ohnehin notleidende Unternehmen finanziell überfordert und gleichzeitig die Akzeptanz dieses noch recht jungen Arbeitsmarktinstruments gefährdet.

Neben den Bedenken bezüglich des Zeitpunkts eines Systemwechsels gibt es auch systemimmanente Faktoren, die zumindest aktuell gegen eine Kopplung des Mindestlohns an $60 \%$ des Medianlohns sprechen. Anders als in den beiden Partnerländern kommt in Deutschland der Tarifautonomie und den Tarifparteien immer noch eine zentrale Rolle zu. Die Tarifautonomie genießt Verfassungsrang und sie wird vom Staat respektiert. Seit Beginn der Bundesrepublik Deutschland haben sich die verschiedenen Bundesregierungen - abgesehen von der Einführung des gesetzlichen Mindestlohns - aus der Tarifautonomie herausgehalten. Zudem sieht sich der deutsche Staat trotz einer rückläufigen Tarifbindung immer noch einem funktionierenden Tarifsystem gegenüber. Auch wenn die Einführung des Mindestlohns tief in die Tarifautonomie eingreift, räumt das MiLoG den Tarifparteien besondere Rechte bei der Anpassung des Mindestlohns ein. Zum einen hat die MLK (und damit Vertreter der Tarifparteien) ein faktisches Vorschlagsmonopol bezüglich bevorstehender Mindestlohnanpassungen. Sie hat mehr Autonomie als in den Vergleichsländern Frankreich und dem Vereinigten Königreich, da die Regierung keine eigenen Erhöhungsvorschläge durchsetzen kann. Zum anderen müssen sich die Beschlüsse der Mindestlohnkommission an der Tariflohnentwicklung orientieren. Diese Regelungen sind nicht zuletzt der Tatsache geschuldet, dass das MiLoG Teil des sogenannten Tarifautonomiestärkungsgesetzes ist, mit dem die Bundesregierung die Tarifautonomie stärken will. Die Aufnahme eines 60-Prozent-Kriteriums würde sowohl die Bedeutung der Tariflohnentwicklung für die Festsetzung der Mindestlohnhöhe relativieren als auch die Autonomie der Tarifpartner in der MLK beschneiden. Beides widerspricht der Intention des Tarifautonomiestärkungsgesetzes.

Ein spürbar steigender Mindestlohn würde deutlich mehr Tariflöhne verdrängen als der bisherige. Dabei besteht die Gefahr, dass in einer ganzen Reihe von Branchen Tarifverhandlungen nicht nur durch den Mindestlohn vorherbestimmt, sondern überflüssig gemacht würden. Um dies zu vermeiden, müsste - wie schon 2015 bei der Mindestlohneinführung - eine Übergangsregelung gewährt werden. Diese Regelung hat sich bewährt und dazu beigetragen, dass der Mindestlohn das Tarifgeschehen in den Niedriglohnbranchen nicht nachhaltig negativ beeinflusst hat.

Im Hinblick auf die Tarifautonomie hat sich auch der bisherige Anpassungsmechanismus bewährt. Negative Effekte auf das Tarifgeschehen waren nur vorüberge- 
hend und vereinzelt zu beobachten, auch die beobachtbaren Stauchungen der Tariflohnstruktur haben nicht dazu geführt, die Verhandlungsbereitschaft zu mindern. Die Tarifparteien haben sich der Aufgabe, die Tariflöhne an die jeweils geltenden Mindestlöhne anzupassen, erfolgreich gestellt. Wenn der Mindestlohn aber schneller steigt als der Tariflohn in arbeitsintensiven Wirtschaftszweigen, wird deren Tariflohndynamik langfristig ganz automatisch immer stärker durch die Mindestlohnentwicklung angetrieben (Lesch und Schröder 2020b, S. 15). Das betrifft nicht nur Branchen, die aktuell Tariflohngruppen auf einem Niveau des gesetzlichen Mindestlohns haben. Langfristig werden auch Branchen getroffen, in denen heute noch ein ausreichender Abstand zum Mindestlohn besteht.

Die Einführung des 60-Prozent-Kriteriums würde sich nicht nur auf das Verhältnis von Staat und Tarifparteien auswirken. Sie würde auch im Widerspruch zur Logik unseres bisherigen Sozialleistungssystems stehen. In Frankreich und im Vereinigten Königreich werden die hohen Mindestlöhne durch Lohnsubventionen flankiert. In Deutschland gibt es eine derartige Unterstützung von Unternehmen bisher nicht. Hier werden geringe Markteinkommen, die den Haushaltsbedarf nicht decken können, im Rahmen einer Bedürftigkeitsprüfung vom Staat aufgestockt, was bei alleinstehenden Vollzeitbeschäftigten schon vor der Einführung des Mindestlohns sehr selten vorkam. Durch einen höheren Mindestlohn könnte dieser Transferbedarf sinken. Das setzt aber voraus, dass die induzierten Lohnsteigerungen keinen negativen Beschäftigungseffekt nach sich ziehen. Die französischen und britischen Erfahrungen zeigen jedoch: Um genau dies zu vermeiden, müsste über die Einführung von Lohnsubventionen nachgedacht werden, die vor allem kleine und mittlere Unternehmen unterstützen. In Frankreich wurden 2019 insgesamt 1,9\% der Lohnkosten oder 1,0\% des Bruttoinlandsprodukts (BIP) für Lohnsubventionen ausgegeben. Das entsprach einer Summe von 23,6 Mrd. €. Die unterschiedliche Art der Gestaltung von Lohnsubventionen zeigt: Der Mindestlohn ist nur eine Stellschraube in einem Gesamtsystem. Verändert man dessen Logik, müssen auch an anderen Stellen Änderungen vorgenommen werden. Der Strategiewechsel im Vereinigten Königreich macht deutlich, welche Folgen die politisch angestrebte Anpassung des MiLoG in Deutschland hätte. Die Einführung eines Living Wage wurde dort explizit als ein Mittel genannt, um von einem System niedriger Löhne und hoher Transfers (mit hohen Steuern und Sozialabgaben) zu einem System hoher Löhne und niedriger Transfers (mit geringen Steuer- und Sozialabgaben) zu gelangen. Darüber sollten sich die politischen Entscheidungsträger, die einen Living Wage propagieren, bewusst werden.

Die Verankerung einer Regel, nach der sich der Mindestlohn neben der Tariflohnentwicklung auch am Medianeinkommen orientieren würde, müsste neben einer Übergangsregelung auch mit einer Korrekturklausel verknüpft werden. Eine solche „Emergency Break“ wurde im Vereinigten Königreich mit der zweiten Stufe des 2016 begonnenen Systemwechsels zu einem Living Wage eingeführt. So kann die LPC bei ökonomischen Problemen oder sonstigen Risiken die Regierung dazu auffordern, ihre Zielvorgaben oder den vorgesehenen Zeitrahmen zu überdenken. Die durch die Corona-Pandemie verursachte Wirtschaftskrise und die Unsicherheit über die wirtschaftlichen Folgen des Brexits haben dazu geführt, dass der nationale Living Wage auf Empfehlung der LPC in diesem Jahr nur um gut $2 \%$ erhöht wurde. 
Diese Erhöhung liegt klar unter dem Zielpfad. Das Ziel, im Jahr 2024 einen Mindestlohn in Höhe von $60 \%$ des Medianlohns der Vollzeitbeschäftigten zu erreichen, wurde indes noch nicht aufgeschoben. Bildlich gesprochen hat die LPC die Hand an die Notbremse gelegt, diese aber noch nicht gezogen. Auch in Frankreich wird über die Flexibilität des Anpassungsprozesses diskutiert. So kritisieren die Mitglieder der wissenschaftlichen SMIC-Expertengruppe den starren Indexierungsmechanismus bezüglich Inflation und allgemeiner Lohnentwicklung. Sie empfehlen seit Jahren, die automatischen Erhöhungsregeln zu reformieren oder ganz abzuschaffen. Sie begründen ihren Vorstoß damit, dass die politischen Autoritäten dadurch mehr Möglichkeiten erhielten, die Mindestlohnerhöhung gemäß der jeweiligen Arbeitsmarktlage auszugestalten (Groupe d'experts sur le SMIC 2020). Diese politischen Diskussionen zeigen: Durch eine verpflichtende Orientierung am Medianeinkommen würde das deutsche System ausgerechnet in einer Phase an Flexibilität einbüßen, in der andere Länder über mehr Flexibilität nachdenken.

Wie die Beispiele von Frankreich und dem Vereinigten Königreich zeigen, ist die Idee eines Living Wage weder neu noch unerprobt. In Deutschland wäre dies ein Systemwechsel, der einen enormen Anpassungsdruck erzeugen würde. Dabei ist zwischen der Zielvorgabe eines Mindestlohns von $12 €$ je Stunde und der Verankerung einer Orientierung des Mindestlohns an 60\% des Medianeinkommens zu unterscheiden. Heute mag der Wert von $12 €$ einem Living Wage von $60 \%$ des Medianeinkommens entsprechen. Bis 2024 wird der Living Wage aber auf 13,20€ steigen und damit deutlich über der Zielmarke von $12 €$ liegen. Geht man bei der nächsten Mindestlohnanpassung im Jahr 2022 von einem Basiswert von 10,45€ aus, würde der Mindestlohn 2023 bei einer nachlaufenden Orientierung am Tarifindex um rund $4 \%$ auf $10,87 €$ je Stunde angehoben. Sollten die Folgen der CoronaPandemie ohne nachhaltige Belastung von Wirtschaft und Arbeitsmarkt bis dahin bewältigt worden sein, könnte für 2024 ein weiterer Zuschlag vereinbart werden, bei dem auch eine erneute ,politische“ Komponente denkbar wäre. Dann läge ein Mindestlohn von $12 €$ zumindest in Reichweite, ohne dass der bisherige Anpassungsmechanismus aufgegeben würde.

Eine Beibehaltung des bewährten und inzwischen eingespielten Anpassungsmechanismus kann dazu beitragen, die politische Auseinandersetzung um den Mindestlohn zu vermeiden. Die MLK hat ihre Autonomie (trotz politischen Drucks) auch bei ihrem letzten Anpassungsbeschluss bewahrt und gleichzeitig der politischen Auseinandersetzung durch einen politisch motivierten Anpassungsschritt auf 10,45€ je Stunde Rechnung getragen. Eine Änderung des MiLoG nähme der Kommission diese Freiheit und sie würde letztlich dazu führen, den Mindestlohn zum politischen Dauerthema zu machen. Angesichts der Schwere des Eingriffs in die Tarifverträge liefe das im Ergebnis auf eine politische Lohnsetzung hinaus. Die Tarifautonomie würde in vielen Branchen ihr Primat gegenüber der Politik einbüßen.

Funding Open Access funding enabled and organized by Projekt DEAL.

Open Access Dieser Artikel wird unter der Creative Commons Namensnennung 4.0 International Lizenz veröffentlicht, welche die Nutzung, Vervielfältigung, Bearbeitung, Verbreitung und Wiedergabe in jeglichem Medium und Format erlaubt, sofern Sie den/die ursprünglichen Autor(en) und die Quelle ord- 
nungsgemäß nennen, einen Link zur Creative Commons Lizenz beifügen und angeben, ob Änderungen vorgenommen wurden.

Die in diesem Artikel enthaltenen Bilder und sonstiges Drittmaterial unterliegen ebenfalls der genannten Creative Commons Lizenz, sofern sich aus der Abbildungslegende nichts anderes ergibt. Sofern das betreffende Material nicht unter der genannten Creative Commons Lizenz steht und die betreffende Handlung nicht nach gesetzlichen Vorschriften erlaubt ist, ist für die oben aufgeführten Weiterverwendungen des Materials die Einwilligung des jeweiligen Rechteinhabers einzuholen.

Weitere Details zur Lizenz entnehmen Sie bitte der Lizenzinformation auf http://creativecommons.org/ licenses/by/4.0/deed.de.

\section{Literatur}

BEIS-Department of Business, Energy \& Industrial Strategy (2020). National living wage and national minimum wage: low pay commission remit 2020. https://assets.publishing.service.gov.uk/ government/uploads/system/uploads/attachment_data/file/871414/nmw-nlw-lpc-remit-2020.pdf. Zugegriffen: 11. Jan. 2021.

Bispinck, R., Dribbusch, H., Kestermann, C., Lesch, H., Lübker, M., Scheider, H., Schröder, C., Schulten, T., \& Vogel, S. (2020). Entwicklung des Tarifgeschehens vor und nach der Einführung des gesetzlichen Mindestlohns. In BMAS (Hrsg.): Forschungsbericht Nr. 562. https://www.bmas.de/SharedDocs/ Downloads/DE/PDF-Publikationen/Forschungsberichte/fb-562-entwicklung-tarifgeschehen-vorund-nach-einfuehrung-mindestlohn.pdf?_blob=publicationFile\&v=3. Zugegriffen: 7. Jan. 2021.

BIS-Department for Business, Innovation and Skills (2015). National minimum wage: interim government evidence for the 2016 low pay commission october 2015. https://assets.publishing.service.gov. uk/government/uploads/system/uploads/attachment_data/file/482910/BIS-15-481-NMW-interimevidence-2015.pdf. Zugegriffen: 9. Jan. 2021.

BMAS - Bundesministerium für Arbeit und Soziales (2021). Eckpunkte zur Weiterentwicklung des Mindestlohns und Stärkung der Tarifbindung, März. https://www.bmas.de/SharedDocs/Downloads/DE/ Arbeitsrecht/fairer-mindestlohn.pdf?_blob=publicationFile\&v=2. Zugegriffen: 29. Apr. 2021.

Bundesagentur für Arbeit (2020). Sozialversicherungspflichtige Bruttoarbeitsentgelte (Jahreszahlen), Deutschland, West/Ost, Länder und Kreis, Stichtag: 31. Dezember 2019, Tabellen.

Cahuc, P., \& Carcillo, S. (2012). Les conséquences des allégements généraux de cortisations patronales sur les bas salaires. Revue Française D'Économie, 27(2), 19-61.

Cette, G., \& Wasmer, E. (2012). Les enjeux des changements de règles de revalorisation automatique du SMIC, LIEPP Working Paper. https://spire.sciencespo.fr/hdl:/2441/10av5r3jd18pmoqdkopa5b5vgl/ resources/wp5-cette-wasmer.pdf. Zugegriffen: 22. Dez. 2020.

Cribb, J., Joyce, R., \& Xu, X. (2019). The future path of minimum wages, IFS Briefing Note BN260. https://www.ifs.org.uk/uploads/BN260-the-future-path-of-minimum-wages.pdf. Zugegriffen: 11. Jan. 2021.

Deutscher Bundestag (2018). Gesetzlicher Mindestlohn in Deutschland und Frankreich: Regelung und Wirkung, Sachstand WD 6-3000-141/18. https://www.bundestag.de/resource/blob/658932/ 4a23b794eb8633d8cc2506ff97c43871/WD-6-141-18-pdf-data.pdf. Zugegriffen: 21. Dez. 2020.

Europäische Kommission (2020). Vorschlag für eine Richtlinie des Europäischen Parlaments und des Rates über angemessene Mindestlöhne in der Europäischen Union vom 28.10.2020, COM(2020) 682 final.

Francis-Devine, B. (2020). National minimum wage statistics, house of commons library briefing paper number 7735. https://researchbriefings.files.parliament.uk/documents/CBP-7735/CBP-7735.pdf. Zugegriffen: 9. Jan. 2021.

Französische Botschaft in Deutschland (2020). Modalitäten des Mindestlohns in Frankreich. https://de. ambafrance.org/Modalitaten-des-Mindestlohns-in. Zugegriffen: 22. Dez. 2020.

Gautié, J., \& Laroche, P. (2018). Minimum wage and the labor market: what can we learn from the French experience? In Documents de travail du Centre d'Economie de la Sorbonne 2018.19.

Groupe d'experts sur le SMIC (2020). Rapport annuel du Groupe d'experts SMIC, 27. November 2020. https://www.tresor.economie.gouv.fr/Articles/284b121f-b187-4280-b327-05f18064c3fa/files/ 962164d8-bc09-457a-be64-86014049c3c6. Zugegriffen: 8. Jan. 2021.

Heil, H. (2020). Die Corona-Krise muss zu höheren Löhnen führen, Interview, Bild am Sonntag, 13.12.2020. https://www.bild.de/bild-plus/politik/inland/politik-inland/hubertus-heil-zu-bams-die- 
corona-krise-muss-zu-hoeheren-loehnen-fuehren-74432284, view=conversionToLogin.bild.html. Zugegriffen: 14. Jan. 2021.

HM Treasury (2015). Summer Budget 2015. https://assets.publishing.service.gov.uk/government/uploads/ system/uploads/attachment_data/file/443232/50325_Summer_Budget_15_Web_Accessible.pdf. Zugegriffen: 9. Jan. 2021.

HM Treasury (2018). Budget 2018. https://assets.publishing.service.gov.uk/government/uploads/system/ uploads/attachment_data/file/752202/Budget_2018_red_web.pdf. Zugegriffen: 11. Jan. 2021.

Lesch, H., \& Schröder, C. (2020a). Auswirkungen des gesetzlichen Mindestlohns. In Mindestlohnkommission (Hrsg.): Stellungnahmen aus der schriftlichen Anhörung. Ergänzungsband zum Dritten Bericht der Mindestlohnkommission an die Bundesregierung nach $\S 9$ Abs. 4 Mindestlohngesetz. https:// www.mindestlohn-kommission.de/DE/Bericht/pdf/Ergaenzungsband-Stellungnahmen2020.pdf? blob=publicationFile \&v=3. Zugegriffen: 8. Jan. 2021.

Lesch, H., \& Schröder, C. (2020b). Zur Höhe des Mindestlohns. APuZ, 70(39-40), 11-15.

Lesch, H., Mayer, A., \& Schmid, L. (2014). Das deutsche Mindestlohngesetz: Eine erste ökonomische Bewertung. List-Forum für Wirtschafts- und Finanzpoliti, 40(1), 1-19.

LPC_Low Pay Commission (2016). National minimum wage: low pay commission report spring 2016. https://assets.publishing.service.gov.uk/government/uploads/system/uploads/attachment_data/file/ 571631/LPC_spring_report_2016.pdf. Zugegriffen: 7. Jan. 2021.

LPC_Low Pay Commission (2019). A review of the youth rates of the national minimum wage november 2019. https://assets.publishing.service.gov.uk/government/uploads/system/uploads/attachment_data/ file/845076/A_Review_of_the_Youth_Rates_of_the_National_Minimum_Wage.pdf. Zugegriffen: 11. Jan. 2021.

LPC_Low Pay Commission (2020). National minimum wage: low pay commission report 2020. https:// assets.publishing.service.gov.uk/government/uploads/system/uploads/attachment_data/file/942062/ LPC_Report_2020.pdf. Zugegriffen: 11. Jan. 2021.

Lübker, M., \& Schulten, T. (2021). WSI-Mindestlohnbericht 2021. Ist Europa auf dem Weg zu angemessenen Mindestlöhnen?. In WSI-Report Nr. 63. https:/www.wsi.de/de/faust-detail.htm?sync_id=HBS007963. Zugegriffen: 29. Apr. 2021.

Mesch, M. (2011). Lohnverhandlungen und gesetzlicher Mindestlohn in Frankreich. Wirtschaft und Gesellschaft, 31(4), 555-588.

MLK - Mindestlohnkommission (2016). Beschluss der Mindestlohnkommission nach $§ 9$ MiLoG. https:// www.mindestlohn-kommission.de/DE/Bericht/pdf/Beschluss2016.pdf?_blob=publicationFile\& v=9. Zugegriffen: 6. Jan. 2021.

MLK - Mindestlohnkommission (2018). Beschluss der Mindestlohnkommission nach $§ 9$ MiLoG. https:// www.mindestlohn-kommission.de/DE/Bericht/pdf/Beschluss2018.pdf?_blob=publicationFile\& v=11. Zugegriffen: 6. Jan. 2021.

MLK - Mindestlohnkommission (2020). Beschluss der Mindestlohnkommission nach § 9 MiLoG. https:// www.mindestlohn-kommission.de/DE/Bericht/pdf/Beschluss2020.pdf?_blob=publicationFile\& $\mathrm{v}=5$. Zugegriffen: 6. Jan. 2021.

OECD - Organisation for Economic Co-operation and Development (1995). OECD economic surveys: France 1995, paris. https://read.oecd-ilibrary.org/economics/oecd-economic-surveys-france-1995_ eco_surveys-fra-1995-en\#page1. Zugegriffen: 3. Mai 2021.

OECD_Organisation for Economic Co-operation and Development (2021). Dataset: minimum relative to average wages of full-time workers. https://stats.oecd.org/Index.aspx?DataSetCode=MIN2AVE. Zugegriffen: 15. Jan. 2021.

Office for Budget Responsibility (2015). Economic and fiscal outlook. Cm 9088, office for budget responsibility - Cm 9088-economic and fiscal outlook july 2015. publishing.service.gov.uk. Zugegriffen: 7. Jan. 2021.

Pusch, T., \& Schulten, T. (2019). Mindestlohn von 12 Euro: Auswirkungen und Perspektiven. Wirtschaftsdienst, 98(5), 335-339.

Riechert, C., \& Nimmerjahn, L. (2017). Mindestlohngesetz (2. Aufl.). München: C.H. Beck.

Schulten, T., \& Lübker, M. (2020). WSI-Mindestlohnbericht 2020: Europäische Mindestlohninitiative vor dem Durchbruch? WSI Mitteilungen, 73(2), 119-129.

Schulten, T., \& Müller, T. (2020). Zwischen Armutslöhnen und Living Wages: Mindestlohnregime in der Europäischen Union, Europäische Studien zur Sozial- und Arbeitsmarktpolitik Band 1. https://www. dielinke-europa.eu/kontext/controllers/document.php/976.9/a/09ed87.pdf. Zugegriffen: 9. Jan. 2021.

Sellers, P. J. (2017). The UK living wage: a trade union perspective. Employee Relations, 39(6), 790-799.

Statistisches Bundesamt (2020). Verdienststrukturerhebung: Niveau, Verteilung und Zusammensetzung der Verdienste und der Arbeitszeiten abhängiger Beschäftigungsverhältnisse - Ergebnisse für 
Deutschland - 2018, Fachserie 16 Heft 1. https://www.destatis.de/DE/Themen/Arbeit/Verdienste/ Verdienste-Verdienstunterschiede/Publikationen/Downloads-Verdienste-und-Verdienstunterschiede/ verdienststrukturerhebung-heft-1-2162001189004.pdf?_blob=publicationFile. Zugegriffen: 14. Jan. 2021.

Statistisches Bundesamt (2019). Entwicklung des Tarifgeschehens vor und nach Einführung des gesetzlichen Mindestlohns. Sonderauswertung für das BMAS, Wiesbaden, 19.09.2019.

Wissenschaftliche Dienste (2016). Die Geschäftsordnung der Mindestlohnkommission. Regelung der Beschlussfassung. https://www.bundestag.de/resource/blob/424060/db8f0fb799b724c08460b882e93d 6ebf/wd-6-041-16-pdf-data.pdf. Zugegriffen: 6. Jan. 2021. 